\title{
EDITORIAL
}

\section{Cellular resistance mechanisms with impact on the therapy of multiple myeloma}

\author{
F Gieseler ${ }^{1}$ and $\vee$ Nußler ${ }^{2}$ \\ ${ }^{1}$ University Hospital and School of Medicine, Medizinische Poliklinik, Wurzburg and ${ }^{2}$ University Hospital, Medizinische Klinik III, Klinikum \\ Großhadern, Munich, Germany
}

\begin{abstract}
Multiple myeloma (MM) is characterized by bone marrow infiltration with abnormal plasma cells which synthesize monoclonal immunoglobulins (Ig) or Ig fragments. Regularly, MM cells exhibit a high intrinsic resistance to available chemotherapeutic strategies. A number of cellular alterations including the cellular membrane, such as mutations of the glucocorticoid receptor or expression of membrane transport proteins, detoxification mechanisms and altered expression of topoisomerases, have been described. In addition to anti-apoptotic survival mechanisms, involving abnormalities of several oncogenes and suppressor genes (ras, c-myc, p53, Rh and bcl-2), the broad resistance spectrum might be explained but clinical studies which include the evaluation of resistance factors are missing. On the other hand, risk factor evaluation is important as a number of therapeutical strategies with different intensities from corticosteroid monotherapy up to high-dose chemotherapy with tandem autologous bone marrow transplantation exist.

Keywords: multiple myeloma; cellular resistance; P-glycoprotein; topoisomerases
\end{abstract}

\section{Introduction}

Despite three decades of clinical trials the number of true complete remissions in the therapy of multiple myeloma (MM) is still low, one of the main reasons being intrinsic or acquired cellular resistance. In this paper the knowledge of cellular resistance mechanisms is reviewed and the impact on therapeutic strategies discussed.

\section{Biological background}

Multiple myeloma is defined by bone marrow infiltration of neoplastic plasma cells that synthesize abnormal amounts of immunoglobulin (Ig) or Ig fragments. The target cell of malignant transformation in multiple myeloma is a B lineage cell which has already undergone antigenic selection. This conclusion can be drawn from the observation that somatic mutations which result in amino acid substitutions are frequent in the Ig variable region genes in $\mathrm{MM}$, but intra-clonal variation has not been shown. ${ }^{1}$ This $B$ lineage cell probably corresponds to a pre-plasma cell or a plasma cell rather than a memory B cell. The myeloma cells are composed of immunophenotypically heterogeneous sub-populations at various stages of differentiation, similar to normal B lineage cells. This implies that there is an analogous developmental pathway between the normal B lineage cells and the myeloma cells. ${ }^{1}$

Correspondence: F Gieseler, University Hospital and Medical School, Medizinische Poliklinik, Klinikstr. 8, 97070 Wurzburg, Germany; Fax: 9312017073

Received 9 October 1997; accepted 12 March 1998

\section{Genetic alterations and oncogenes}

Cytogenetic alterations are frequent in MM. In as many as $30 \%$ of patients hyper-diploidy and in 15\% hypo-diploidy has been observed in a cytogenetic study of 151 patients. The most frequent numeric change was the loss of chromosome 13 which occurred in $15 \%$ of patients. ${ }^{2}$

The oncogenes bcl-2 and $\mathrm{H}$-ras seem to play exceptional roles in the transformation process of myeloma cells. Overexpression of the bcl-2 protein has been almost uniformly seen in clinical myeloma specimens as well as in myeloma cell lines. The failure to consistently identify a t $(14 ; 18)$ translocation implies a unique mechanism in $\mathrm{MM}^{3} \mathrm{~T}(14 ; 18)$ translocations are frequently found in follicular lymphomas and are characteristically associated with over-expression of bcl-2, which has been linked to the inhibition of apoptotic response to chemotherapy. ${ }^{4,5}$

Mutated alleles of ras genes have been detected in a high percentage of myeloma patients in relapse phase. ${ }^{6,7} \mathrm{~A}$ point mutated and activated $\mathrm{H}$-ras oncogene, introduced in a human lymphoblastoid cell line, was able to induce neoplastic transformation and differentiation to plasma cells. This indicates a link between the ras mutations and the transformation to myeloma cells. ${ }^{7}$ Ras mutations have been associated with cellular resistance to a number of cytostatics including doxorubicin and arabinoside..$^{8,9}$

\section{Cytokines and growth factors}

Excessive osteoclastic resumption is an early phenomenon of osteolytic bone lesions found in $\mathrm{MM}$ and a number of cytokines and growth factors such as IL-6, TNF, IL-1 beta, G-CSF and GM-CSF are probably involved. ${ }^{10}$ Interleukin-6 (IL-6) seems to be of special importance for the generation of osteolytic bone lesion in MM. IL-6 is a multi-functional cytokine involved in the regulation of the terminal differentiation pathway of B lymphocytes. Thus, IL- 6 is a potent myeloma cell growth factor. ${ }^{11}$ There is some evidence that IL-6 influences progression of $\mathrm{MM}$ in an autocrine or paracrine manner and IL-6 serum levels are in correlation with disease activity, prognosis and survival. ${ }^{12-15} \mathrm{IL}-1$ and TNF are able to induce IL-6 secretion by osteoclasts which stimulates the generation of new osteoclasts and bone resorption. ${ }^{16}$ This cytokine network indicates a close relationship between MM cell stimulation and the induction of osteolytic bone lesions.

Besides IL-6, IFN-alpha is also a potent myeloma cell growth factor in vitro. It has been shown to stimulate the proliferation of most IL-6-dependent myeloma cell lines. IFNalpha can be used to induce autocrine production of IL-6 in myeloma cells and autonomously growing cell lines can be produced very quickly. ${ }^{16} \mathrm{IFN}$-alpha is able to inhibit the production of the monoclonal component without affecting pro- 
Table 1 Expression of $\mathrm{P}$-gp in 25 patients with $\mathrm{MM}$ at primary diagnosis in relation to clinical features by using the indirect immunocytological method with mAb 4.E3.16

\begin{tabular}{lcc}
\hline & $\begin{array}{c}\text { No. of P-gp- } \\
\text { positive patients }\end{array}$ & $\begin{array}{c}\text { No. of P-gp- } \\
\text { negative patients }\end{array}$ \\
\hline Total & $8(32 \%)$ & $17(68 \%)$ \\
Stage & 2 & 5 \\
I & 2 & 7 \\
II & 4 & 5 \\
III & 2 & 9 \\
Ig A & 5 & 4 \\
Paraprotein & 1 & 4 \\
Serum $\beta_{2}$-microglobulin & 2 & 11 \\
$>2.5$ mg/l & 5 & 6 \\
$<2.5$ mg/l & 3 & \\
\hline
\end{tabular}

liferation. ${ }^{17}$ Therefore, IFN-alpha might mask an early relapse and the continuous injection of IFN-alpha in patients in early relapse might induce myeloma sub-clones with autocrine IL6 production and worsen the disease. ${ }^{16}$ IFN-gamma, on the other hand, acts as a potent inhibitor of myeloma cell proliferation by blocking the IL-6 receptor expression. A study by Portier et $a l^{18}$ has also shown that IFN-gamma is able to inhibit cytokine-mediated bone resorption.

\section{Steroid-receptor alterations}

Despite their widespread use, little is known of either the mechanism of action of glucocorticoids in the treatment of multiple myeloma or why patients ultimately become resistant to their therapeutic effects. Moalli et a ${ }^{19}$ have shown that resistance to dexamethasone can be induced in myeloma cell lines. The difference in the sensitive lines was the expression of a truncated glucocorticoid receptor mRNA resulting in a greatly reduced hormone-binding activity. The significance of this observation in clinical studies has not been examined.

\section{P170-glycoprotein}

Expression of the transmembrane P170-glycoprotein (P-gp) in $\mathrm{MM}$ has been investigated by several groups. ${ }^{20-27}$ The percentage of patients with P-gp overexpression at primary diagnosis ranged between 0 and $41 \% .^{20-23}$ The percentage of $\mathrm{P}_{-}$ gp-positive patients at relapse or progression ranged between 29 and $59 \% .^{24,25}$ In our study $32 \%$ of the patients at primary diagnosis (Table 1 ) and $33 \%$ of patients at relapse or progression were P-gp positive (Table 2). (In our study we found

Table 2 MM patients at relapse or progression according to response to VAD treatment and P-gp positivity by using the indirect immunocytological method with mAb 4.E3.16

\begin{tabular}{lc}
\hline & No. of patients (\%) \\
\hline Response/Total & $5 / 18(28)$ \\
P-gp positive/Total & $6 / 18(33)$ \\
Response/P-gp positive & $2 / 6(33)$ \\
P-gp negative/Total & $12 / 18(67)$ \\
Response/P-gp negative & $5 / 12(42)$ \\
\hline
\end{tabular}

a positivity in Table 2.) Six of 12 (50\%) P-gp-negative MM patients at relapse or progression before VAD therapy developed P-gp overexpression after this regimen. All six MM patients did not respond, four patients demonstrated no change and two patients showed progression. No significant difference concerning survival time betgween P-gp-positive (median 25.4 months) and P-gp-negative (median 29.6 months) MM patients was seen.

These data suggest that P-gp overexpression does not influence response to treatment significantly. The assumption that P-gp might be a crucial resistance mechanism was based upon early reports $20,28,29$ and reactivated by the fact that prior exposure to vincristine and doxorubicin induces an increase in P-gp expression in human plasma cell myelomas. ${ }^{22}$ Additionally, Sonneveld et $a^{21}$ demonstrated that cyclosporine, a so-called modulator of P-gp, has the potency to overcome chemotherapy refractoriness in MM patients. However, no relationship between P-gp expression and response to VAD treatment ${ }^{24,30}$ and no benefit from the modulator verapamil could be shown in larger trials. ${ }^{30}$ On the other hand, the results of several studies indicate that P-gp modulators are able to restore the sensitivity of doxorubicin. ${ }^{31-35}$

For the interpretation of these conflicting results a number of considerations must be taken into account. All treatment protocols including VAD (vincristine, doxorubicin and dexamethasone), VID (vincristine, idarubicin and dexamethasone) and VMD (vincristine, mitoxantrone and dexamethasone) contain corticosteroids which are highly effective drugs but not transported by P-gp. Moreover, no standardized methods are available which clearly define the amount of mdr1 gene expression or P-gp protein expression necessary to establish clinical relevance.

\section{Glutathione S-transferase}

Glutathione is involved in many biological functions including detoxification of alkylating agents and platinum complexes. Also, its activity has been connected to cellular melphalan resistance. ${ }^{36}$ In a study by Ishikawa et a/ ${ }^{3} 16$ of 21 (76\%) myeloma cases showed significant expression of GSTpi protein and GST-pi mRNA. In another study, which included bone marrow samples from 40 patients, $72 \%$ of evaluated samples were positive for P-170 glycoprotein and $82 \%$ for GST-pi. A significant relationship between GST-pi expression and P-170 positivity was found and co-expression was observed in $91 \%$ of evaluated samples with an increase in relapse. ${ }^{6}$

\section{Topoisomerase}

Most of the resistant myeloma cell lines which are used for in vitro examination of resistance mechanisms have been produced by increasing concentrations of the selecting agent over a long period of time. This procedure does not reflect the clinical situation where chemotherapy is given in a repetitive schedule. Likely, the evolving cellular resistance mechanisms depend upon the selection method. In a co-operative study with Lehnert et $a^{\beta 7}$ from the Kantonspital in St Gallen, Switzerland, resistant myeloma cell lines using a quasi-therapeutical schedule have been selected. The sensitive myeloma cell line has been incubated with $5 \mathrm{~nm}$ or $10 \mathrm{~nm}$ doxorubicin for 4 days and then kept without drug for 17 days. This procedure was repeated for 18 months. Two resistant cell lines, 
Table 3 Resistance factors of myeloma cell lines $\left(\mathrm{IC}_{50}\right.$ selected cell line/ $/ C_{50}$ S-8226)

\begin{tabular}{lccccc}
\hline \multirow{2}{*}{ Drugs } & \multicolumn{2}{c}{ Dox-int5 } & & \multicolumn{2}{c}{ Dox-int10 } \\
\cline { 2 - 3 } \cline { 5 - 6 } & -Verapamil & +Verapamil & & -Verapamil & +Verapamil \\
\hline Doxorubicin & 3.8 & 3.2 & & 4.8 & 4.3 \\
Etoposide & 4.2 & 3.6 & & 6.0 & 5.1 \\
Amsacrine & 3.3 & 2.3 & & 3.2 & 2.3 \\
Vincristine & 1.6 & 1.0 & & 1.8 & 1.0 \\
Paclitaxel & 1.2 & 1.1 & & 1.2 & 0.8 \\
Melphalan & 1.1 & - & & 1.1 & - \\
Cisplatin & 1.2 & - & & 1.1 & - \\
\hline
\end{tabular}

Verapamil concentration was $5 \mu \mathrm{M}$.

named Dox-int5 and Dox-int10, emerged that showed a stable resistance for at least 6 months.

As shown in Table 3, the resulting resistance factors were not as high as in cell lines after continuous incubation with a predominance of topo-II-inhibitors and low resistance to melphalan or cisplatin. The differences between the dox-int5 cell line and the dox-int10 cell line were not high. In contrast to resistant cell lines selected by continuous exposure, neither MDR1 mRNA nor P170-glycoprotein expression were elevated as compared to the sensitive line, which can also be suspected by the low effect of verapamil on resistance. Topo-II alpha gene expression and activity was notably lower in both cell lines which might well explain the resistance to topo-II. ${ }^{37}$ In clinical material, expression of topo-II mRNA was detected weakly in only one of 16 myeloma patients. ${ }^{23}$

\section{Possible clinical relevance}

A characteristic feature of MM cells is their intrinsic resistance to available treatment modalities. Likely, the resistance is a consequence of an array of anti-apoptotic survival mechanisms involving abnormalities of several oncogenes and suppressor genes (ras, c-myc, p53, Rb, and bcl-2) in combination with cellular alterations which affect all cellular levels (Figure 1). These include the cellular membrane, such as mutations of the glucocorticoid receptor or expression of membrane transport proteins, detoxification mechanisms and altered expression of topoisomerases. Whereas anti-apoptotic mechanisms affect the majority of chemotherapeutic strategies

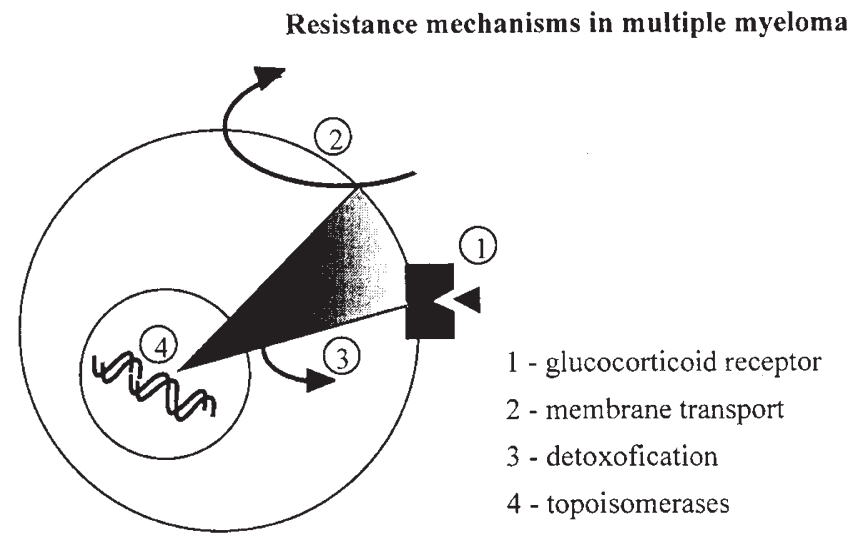

Figure 1 Cellular resistance mechanisms in multiple myeloma.
Editorial

F Gieseler and V Nußler

resulting in a broad resistance spectrum, specific cellular resistance mechanisms might result in high resistance to certain drugs. However, the clinical relevance of the described resistance mechanisms cannot be weighted conclusively due to missing clinical studies which include evaluation resistance factors. On the other hand, risk factor evaluation is of significance for the patient as a number of therapeutical strategies from corticosteroid monotherapy up to high-dose chemotherapy with tandem autologous bone marrow transplantation exist.

Up to now it has been shown that certain cytogenetic abnormalities such as partial or complete deletion of chromosome 13 or abnormalities involving $11 \mathrm{q}^{38}$ as well as proliferation-associated markers are coupled with poor outcome. Creactive protein (CRP $\leqslant 4 \mathrm{mg} / \mathrm{l})$, which reflects IL-6 activity, beta-2-microglobulin $\left(\beta_{2} M \leqslant 2.5 \mathrm{mg} / \mathrm{l}\right)$, in addition to no more than 1 year of prior therapy, presence of sensitive disease $(>50 \%$ tumor mass reduction prior to transplant), and lactic dehydrogenase levels not exceeding $190 \mathrm{U} / \mathrm{l}$ are all independent favourable prognostic factors for event-free survival and overall survival. ${ }^{39}$ Most of the studies presented concerning cellular resistance mechanisms have been performed using myeloma cell lines because in the majority of patient bone marrow infiltration by MM is low and the cells must either be selected or stimulated in vitro. Except for the study of Lehnert et $a^{\beta 7}$ resistant myeloma cell lines have been selected using increasing concentrations of the selecting agent over a long period of time. This procedure alters cellular biology and these in vitro results cannot be directly transferred into the clinical situation. For the development of individual riskadapted therapeutical strategies clinical studies which include the assessment of cellular resistance factors in clinical material are required.

\section{References}

1 Takishita M, Kosaka M. Multiple myeloma: new evidence and insights from the immunoglobulin heavy chain gene and phenotypes. Leuk Lymphoma 1995; 19: 395-400.

2 Lai JL, Zandecki M, Mary JY, Bernardi F, Izydorczyk V, Flactif M, Morel P, Jouet JP, Bauters F, Facon T. Improved cytogenetics in multiple myeloma: a study of 151 patients including 117 paients at diagnosis. Blood 1995; 85: 2490-2497.

3 Niesvizky R, Siegel D, Michaeli J. Biology and treatment of multiple myeloma. Blood Rev 1993; 7: 24-33.

4 Lotem J, Sachs L. Regulation by bcl-2, c-myc, and p53 of susceptibility to induction of apoptosis by heat shock and cancer chemotherapy compounds in differentiation-competent and -defective myeloid leukemic cells. Cell Growth Differ 1993; 4: 41-47.

5 Kamesaki S, Kamesaki H, Jorgensen TJ, Tanizawa A, Pommier Y, Cossman J. bcl-2 protein inhibits etoposide-induced apoptosis through its effects on events subsequent to topoisomerese IIinduced DNA strand breaks and their repair. Cancer Res 1993; 53: 4251-4256.

6 Petrini M, Di Simone D, Favati A, Mattii L, Valentini P, Grassi B. GST-pi and P-170 co-expression in multiple myeoma. Br J Haematol 1995; 90: 393-397.

7 Pileri A, Ferrero D, Massaia M, Dianzani U,Boccadoro M. Advances in biology of multiple myeloma: cell kinetics, molecular biology and immunology. Eur J Haematol Suppl 1989; 51: 30-34.

8 Nooter K, Boersma AW, Oostrum RG, Burger H, Jochemsen AG, Stoter G. Constitutive expression of the C-H-ras oncogene inhibits doxorubicin-induced apoptosis and promotes cell survival in a rhabdomyosarcoma cell line. Br J Cancer 1995; 71: 556-561.

9 Riva C, el Khyari S, Rustum Y, Barra Y. Resistance to cytosine arabinoside in cells transfected with activated Ha-ras oncogene. Anticancer Res 1995; 15: 1297-1302.

10 Bataille R, Klein B. Mechanisms of bone lesions in multiple myeloma. Hematol/Oncol Clin North Am 1992; 6: 285-295. 
11 Zhang XG, Klein B, Bataille R. Interleukin-6 is a potent myelomacell growth factor in patients with aggressive multiple myeloma. Blood 1989; 74: 11-13.

12 Kyle RA. Prognostic factors in multiple myeloma. Stem Cells 1995; 13 (Suppl. 2): 56-63.

13 Reibnegger G, Krainer M, Herold M, Ludwig H, Wachter H, Huber $H$. Predictive value of interleukin- 6 and neopterin in patients with multiple myeloma. Cancer Res 1991; 51: 6250-6253.

14 Kyrstsonis MC, Dedoussis G, Baxevanis C, Stamatelou M, Maniatis A. Serum interleukin-6 (IL-6) and interleukin-4 (IL-4) in patients with multiple myeloma (MM). Br J Haematol 1996; 92: 420-422.

15 Cimino G, Avvisati G, Amadori S, Cava MC, Giannarelli D, Di Nucci GD, Magliocca V, Petrucci MT, Poti G, Sgadari C et al. High serum IL-2 levels are predictive of prolonged survival in multiple myeloma. Br J Haematol 1990; 75: 373-377.

16 Klein B. Cytokine, cytokine receptors, transduction signals, and oncogenes in human multiple myeloma. Semin Hematol 1995; 32: 4-19.

17 Tanaka H, Tanabe O, Iwato K, Asaoku H, Ishikawa H, Nobuyoshi M, Kawano M, Kuramoto A. Sensitive inhibitory effect of interferon-alpha on M-protein secretion of human myeloma cells. Blood 1989; 74: 1718-1722.

18 Portier M, Zhang X, Ursule E. Cytokine gene expression in human multiple myeloma. Blood 1993; 81: 3076-3082.

19 Moalli PA, Pillay S, Weiner D, Leikin R, Rosen ST. A mechanism of resistance to glucocorticoids in multiple myeloma: transient expression of a truncated glucocorticoid receptor mRNA. Blood 1992; 79: 213-222.

20 Riccardi A, Invernizzi R, Ucci G et al. P170 glycoprotein in multiple and acute leukemia. Haematologica 1991; 76 (Suppl.): 177-180.

21 Sonneveld P, Durie G, Lokhorst HM et al. Modulation of multidrug-resistant multiple myeloma by cyclosporine. Lancet 1992; 340: 255-259.

22 Grogan TM, Spier CM, Salmon SE et al. P-glycoprotein expression in human plasma cell myeloma: correlation with prior chemotherapy. Blood 1993; 81: 490-495.

23 Ishikawa H, Kawano MM, Okada K, Tanaka H, Tanabe O, Sakai A, Asaoku H, Iwato K, Nobuyoshi M, Kuramoto A. Expression of DNA topoisomerase I and II gene and the genes possibly related to drug resistance in human myeloma cells. Br J Haematol 1993; 83: $68-74$

24 Cornelissen JJ, Sonneveld P, Schoester M et al. MDR-1 expression and response to vincristine, doxorubicin and dexamethasone chemotherapy in multiple myeloma refractory to alkylating agents. J Clin Oncol 1991; 12: 115-119.

25 Patriarca F, Melli C, Damiani D et al. Plasma cell p170 expression and response to treatment in multiple myeloma. Haematologica 1996; 81: 232-237.

26 Ucci G, Petrini M, Riccardi A et al. Expression of p170 protein in multiple myeloma: a clinical study. Hematol Oncol 1992; 10: 213-220.

27 Pilarski LM, Belch AR. Intrinsic expression of the multidrug transporter, P-glycoprotein 170, in multiple myeloma: implications for treatment. Leuk Lymphoma 1994; 17: 367-374.

28 Linsenmeyer $\mathrm{ME}$, Jefferson $\mathrm{S}$, Wolf $\mathrm{M}$ et al. Levels of expression of the mdr1 gene and glutathion-S-transferase genes 2 and 3 and response to chemotherapy in multiple myeloma. Br J Cancer 1992; 65: 471-475.

29 Dalton WS, Durie BGM, Alberts DS et al. Characterization of a new drug-resistant human myeloma cell line that expresses P-glycoprotein. Cancer Res 1986; 46: 5125-5130.

30 Dalton WS. A phase III randomized study of oral verapamil to reverse drug resistance in patients with refractory myeloma. A Southwest Oncology Group Study. Cancer 1994; 75: 815-820.

31 Salmon SE, Dalton SW, Grogan TM et al. Multidrug-resistant myeloma: laboratory and clinical effects of verapamil as a chemosensitizer. Blood 1991; 78: 44-50.

32 Durie B, Dalton WS. Reversal of drug-resistance in multiple myeloma with verapamil. Br J Haematol 1988; 68: 203-206.

33 Solary E, Bidan JM, Calvo F et al. P-glycoprotein expression and in vitro reversion of doxorubicin resistance by verapamil in clinical specimens from acute leukemia and lymphoma. Leukemia 1991; 5: 592-597.

34 Pilarski L, Belch AR. Circulating monoclonal B cells expressing Pglycoprotein may be a reservoir of multidrug resistant disease in multiple myeloma. Blood 1994; 83: 724-736.

35 Dalton WS, Grogan TM, Rybski JA et al. Immunohistochemical detection and quantitation of P-glycoprotein in multiple drugresistance human myeloma cells: association with level of drug resistance and drug accumulation. Blood 1989; 73: 747-752.

36 Medh RD, Gupta V, Awasthi YC. Reversal of melphalan resistance in vivo and in vitro by modulation of glutathione metabolism. Biochem Pharmacol 1991; 42: 439-441.

37 Lehnert M, Wyler B, Shao Y, Schneider E, Cianfriglia M, Scheper R, Frey B, Gieseler F, Schmid L, Twentyman P. Intermittent exposure to doxorubicin treatment in vitro selects for multifactorial non-P-glycoprotein-associated resistance in RPMI 8226 myeloma cells. Br J Haematol 1997; 97: 65-75.

38 Tricto G, Sawyer J, Jagannath S. Poor prognosis in multiple myeloma is associated only with partial or complete deletion of chromosome 13 or abnormalities involving $11 \mathrm{q}$ and not with other karyotype abnormalities. Blood 1995; 86: 4250-4256.

39 Barlogie B. Advances in multiple myeloma treatment: biology and therapy. ASCO Educational Book 1997; 33: 123-134. 